\title{
Preventive Control Strategy of Cascading Fault considering Safety and Economy
}

\author{
Hui-Qiong Deng $\mathbb{D},,^{1,2}$ Jie Luo $\mathbb{D}^{1,2}$ Chen-Chen Li, ${ }^{3}$ Pei-Qiang Li, ${ }^{1,2}$ and Rong-Jin Zheng ${ }^{1,2}$ \\ ${ }^{1}$ School of Electronic, Electrical Engineering and Physics, Fujian University of Technology, Fuzhou 350118, China \\ ${ }^{2}$ Fujian Provincial University Engineering Research Center for Simulation Analysis and Integrated Control of Smart Grid, \\ Fuzhou, Fujian, China \\ ${ }^{3}$ School of Design, Fujian University of Technology, Fuzhou 350118, China \\ Correspondence should be addressed to Hui-Qiong Deng; 1123233466@qq.com
}

Received 30 August 2021; Revised 17 September 2021; Accepted 18 September 2021; Published 11 October 2021

Academic Editor: Yang Li

Copyright (C) 2021 Hui-Qiong Deng et al. This is an open access article distributed under the Creative Commons Attribution License, which permits unrestricted use, distribution, and reproduction in any medium, provided the original work is properly cited.

\begin{abstract}
The operation and structure of the power system are becoming increasingly complex, and the probability of cascading fault increases. To this end, this paper proposes a cascading fault preventive control strategy that considers safety and the economy. First is to give a mathematical form to discriminate the cascading fault according to the action characteristics of the current-type backup protection. Second, the safety and economy of the system are evaluated in terms of power grid safety margin and generation operation cost, respectively, the initial faults are selected based on the power grid vulnerability and safety margin, and a cascading fault preventive control model is constructed for different initial faults' scenarios. The model is a two-layer optimization mathematical model, with the inner model being solved by particle swarm optimization to minimize the power grid safety margin. The outer model is solved by the multiobjective algorithm to minimize generation cost and maximizing power grid safety margin. Finally, the calculated Pareto set is evaluated using fuzzy set theory to determine the optimal generator output strategy. The feasibility of the proposed method is verified by conducting a simulation study with the IEEE39 node system as an example.
\end{abstract}

\section{Introduction}

The modern power grid has evolved toward a highly intelligent, complex, and high-capacity Internet trend, promoting rapid social and economic development along with a series of fault problems. A fault in a small area can quickly involve the entire power grid and then develop into a major blackout accident. Therefore, learning the lessons from accidents, studying the chain fault development process, and formulating sound cascading fault defense decisions have important application values to enhance the safety and reliability of power grid operation.

At the present stage, the most notable studies on modeling aspects of cascading faults are related models based on self-organized criticality theory [1-7], such as the OPA model based on power system weak links $[1,2]$, CASCADE model based on component cascading failure
[3, 4], and Manchester model based on AC-DC currents [5-7]. These models explore the intrinsic mechanism of large outage accidents triggered by cascading faults and reveal that large outage accidents have self-organized critical characteristics, which are of great reference value for preventing cascading faults.

Numerous studies have shown that, in the early stages of cascading faults, the action of relay protection and power flow redistribution are the main causes of further deterioration of the grid operation. To prevent cascading faults, literature [8] proposed a vulnerability-based cascading fault preventive control model, which quantifies the risk of initial faults leading to subsequent faults and improves the safety of power system operation. Literature [9] establishes a Bayesian network-based chain fault model to minimize fault risk and achieve preventive control using machine and load cutting. Literature [10] proposes a multiobjective algorithm-based 
chain trip prevention strategy by screening sensitive control nodes with the goal of less equipment adjustment and less load cutting. In literature [11], multistage dynamic game theory is introduced to analyze the process of a complex system cascading faults and give a risk assessment method of cascading fault accident chain to determine the best preventive control scheme. Literature $[12,13]$ established a coordinated control model for cascading faults considering safety and economy and obtained generation output adjustment and load cutting strategies by solving them. Although the above studies have good reference value for cascading fault analysis and prevention, most of these studies are based on accident chain control methods, which are computationally intensive and time-consuming, can hardly reflect the actual development of cascading faults, and may not be used to guide cascading fault prevention in practice.

Based on the above analysis, this paper mainly focuses on the trigger phase of cascading faults, reflects the safety of the power grid by the distance between the nodal injection power in the current power grid operation state and the nodal injection power in the critical state, reflects the economy of the power grid by the generation operation cost, and establishes a cascading fault preventive control model for different initial faults based on the selection of initial faults using power grid vulnerability and safety margin. For this model, the Pareto solution set under the model is calculated by combining particle swarm algorithm and multiobjective algorithm, and the Pareto solution set is evaluated using fuzzy set theory to determine the optimal generator output strategy. The simulation analysis of the IEEE39 node system is carried out to verify the effectiveness of the method proposed in this paper.

\section{Mathematical Expression of Cascading Fault}

In the event of a blackout, the cascading fault of the power grid is usually caused by the backup protection action caused by the power flow transfer, which usually occurs in the early stage of the blackout. To facilitate the analysis, this paper mainly studies a common cascading fault mode: after the initial fault branch is cut off, with the power flow transfer, the cascading fault occurs due to the corresponding electrical quantity of the remaining branches entering the action area of the backup protection (mainly current protection and distance protection (Section 3)). In a cascading fault sequence, if the initial fault is regarded as the first-level fault, the cascading trip after the initial fault is cut off can be regarded as the second-level fault, the secondlevel fault may also cause the third level fault, and so on. This paper only studies the second-level fault and considers it together with the initial fault.

In this paper, taking each branch configured with current-type backup protection as an example, assuming that, in a certain power grid, after the initial fault of branch $L_{\mathrm{a}}$ is cut off by the backup protection device at a certain time, whether any other branch $L_{b}$ will have cascading fault can be judged according to whether the current flow detected by the configured current protection enters the protection action area. Let the branch $L_{b}$ be between node $i$ and node $j$, and its i-side is configured with current-type backup protection; according to the protection calibration value and measured current, the equation is shown in formula (1):

$$
I_{i . \text { dist }}^{(b)}=\left|I_{i}^{b . \mathrm{set}}\right|-\left|I_{i}^{b . m}\right| \text {, }
$$

where $I_{i}^{b . m}$ is the current measured by i-side backup protection of branch $L_{b}, I_{i}^{b . s e t}$ is the setting value of i-side backup protection of branch $L_{b}$, and $I_{i \text { dist }}^{(b)}$ is the distance between $I_{i}^{b . s e t}$ and $I_{i}^{b . m}$. It can be seen from the specific performance of branch cascading fault that when $I_{i \text {.dist }}^{(b)}>0$, branch $L_{b}$ will not have cascading fault; when $I_{i \text {.dist }}^{(b)}<0$, branch $L_{b}$ will have cascading fault; when $I_{i \text {.dist }}^{(b)}=0$, branch $L_{b}$ is just at the boundary of cascading fault. Similarly, if the j-side of branch $L_{b}$ is also equipped with current protection, just change the superscript from $i$ to $j$.

Let the number of branches $L_{b}$ in the power grid be $b$, and there are $l$ remaining branches except for the initial fault. Considering all the remaining branches, we can further give the expression shown in the following formula:

$$
\left\{\begin{array}{l}
I_{b}=\min \left(I_{i . \text { dist }}^{(b)}, I_{j \text {.dist }}^{(b)}\right) \\
I=\min \left(I_{b}\right) \quad(b=1,2, \ldots, l)
\end{array} .\right.
$$

It can be seen from formula (2) that, from the power grid level, when $I<0$, the power grid is in the cascading fault state, when $I>0$, the power grid is in the safe state, and when $I=0$, the power grid is just in the critical state of cascading fault. In this way, according to formula (2), the mathematical expression for judging the cascading fault of the power grid is given.

\section{The Basic Model of Preventive Control}

Preventive control is usually a control measure before faults occur when the initial fault has not yet occurred and the power grid dispatcher has enough time to adjust the current power grid operation to a safer operating state. Therefore, for the power grid interlocking fault scenario, it will be the problem studied in this paper to establish a preventive control model to ensure the improvement of power grid safety while taking proper care of the power grid economy.

3.1. System Safety. In the triggering stage of cascading fault, the injected power of power grid nodes before and after initial fault removal is unchanged. Therefore, for a given power grid and initial fault, a method for analyzing the safety of the power grid for cascading fault can be given according to the nodal injection power [14].

Assume that the node power combination consists of active power and reactive power and the nodal injection power combination for which no cascading fault occurs is called the safe combination; the nodal injection power combination for which a cascading fault can be triggered is called the dangerous combination. When the power system operates normally, the farther the nodal injection power combination of the current power grid is from the dangerous combination, the higher the safety level of the power grid for cascading faults after the initial fault occurs is. If the shortest distance between the nodal injection power combination and the dangerous combination of the current power grid can be analyzed before the initial fault 
occurs, the safety of the power grid and the allowable variation range of the nodal injection power of the power grid can be observed. For this purpose, Figure 1 gives a further explanation.

In Figure 1, the nodal injection power combination $(\boldsymbol{P}$, $Q)$ is denoted by $S$. $S_{0}$ is the nodal injection power combination corresponding to the current power grid operation state (safety combination), denoted by ', while the nodal injection power combination that can trigger cascading fault (dangerous combination) is denoted by ${ }^{*} ; S_{1}$ is the nodal injection power combination closest to $S_{0}$ in the dangerous combination. With $S_{0}$ being the center of the sphere, the distance between $S_{0}$ and $S_{1}$ is used as the radius to form a circular sphere $A$, and the area outside the sphere is denoted by $B$. From Figure 1 and the previous analysis, it is clear that, for a certain initial fault, no cascading fault occurs when the power grid is operated at any position within sphere $A$. If we can manage to calculate the radius of sphere $A$, we can observe a plausible safety region. Therefore, the radius $R$ of the sphere $A$ can be used to measure the current safety level of the grid for interlocking faults, and $R$ can be expressed as

$$
R=\sqrt{\left|\mathbf{S}_{0}-\mathbf{S}_{1}\right|^{2}}
$$

where $R$ is the distance between $S_{0}$ and $S_{1}$. Furthermore, if it is extended to a multinode power system, formula (3) can be written in the form shown in the following formula:

$$
R=\min \left(\sqrt{\sum_{d=1}^{D}\left|\mathbf{S}_{0 . d}-\mathbf{S}_{1 . d}\right|^{2}}\right),
$$

where $D$ is the total number of nodes of the system; $R$ can be used to characterize the safety margin of the power grid. The greater the $R$ value is, the safer the power grid will be. Therefore, this paper takes it as the safety index of the system.

3.2. System Economy. In this paper, the power generation operation cost is defined as the economic index of the system, and the power generation operation cost $C$ can be expressed as [15]

$$
C=\sum_{u=1}^{\mathrm{GE}}\left(a_{u} P_{\mathrm{Gu}}^{2}+b_{u} P_{\mathrm{Gu}}+c_{u}\right),
$$

where GE is the set of generators participating in the adjustment; $P_{G u}$ is the active output of the generator at node $u$; $a_{u}, b_{u}$, and $c_{u}$ are the operating cost coefficients.

3.3. Mathematical Model of Preventive Control. In this paper, the power output of the generator set is adjusted as a preventive control measure, the control variable is the nodal injection power of the generator, and the interlocking fault prevention control model considering safety and economy is established. The model can be divided into two levels (called inner layer and outer layer, respectively). Among them, the inner model mainly calculates the power grid safety margin, and the outer model optimizes the output of the generator set under the consideration of power grid safety margin and power generation operation cost.

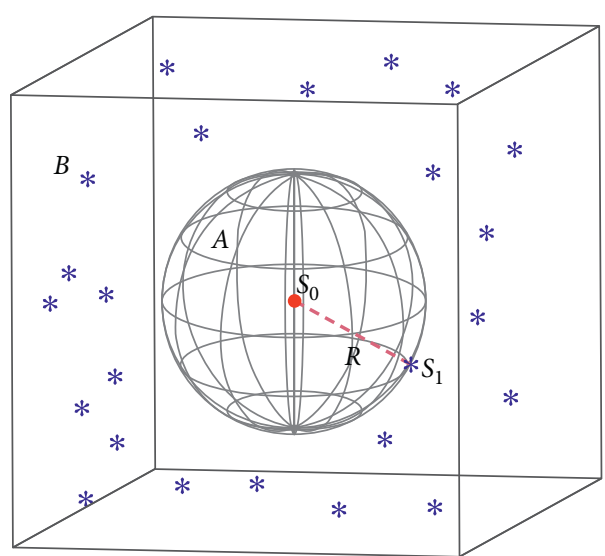

FIgURE 1: Safety level of the power grid for cascading failure.

3.3.1. Inner Layer Optimization Model. The objective function of inner layer optimization is to minimize the safety margin of the power grid, to characterize the shortest distance between the initial operational state of the power grid and the critical state of cascading fault. The objective function $F_{1}$ is as follows:

$$
F_{1}=\min (R) .
$$

Constraints:

(1) Critical constraints of the power grid

To search the critical state closest to the current operation state of the power grid, the cascading fault criticality constraint needs to be introduced. According to the analysis of formulas (1) and (2), under the action of $S$, when the initial fault $L_{a}$ is cut off, the conditions under which the power grid is just in the critical state of cascading fault are as follows:

$$
I=\min \left(I_{b}\right)=0 .
$$

(2) Power flow constraints

The power grid also needs to ensure that the power flow constraints are satisfied before and after the fault in the cascading fault critical state. Among them, the equation constraint is the system node power flow constraint, while the inequality constraint includes generator active and reactive power output constraint, node voltage constraint, and branch transmission power constraint. For the convenience of description, they can be abbreviated to the form shown in the following formula:

$$
\begin{cases}\text { s.t. } & m^{0}(\mathbf{x})=0 \\ & m^{1}(\mathbf{x})=0 \\ & g^{0}(\mathbf{x}) \geq 0 \\ & g^{1}(\mathbf{x}) \geq 0\end{cases}
$$

where $m^{0}$ and $m^{1}$ are the reduced form of the power flow equation constraint corresponding to the power grid before and after the initial fault, respectively; $g^{0}$ and $g^{1}$ are the 
reduced form of the power flow inequality constraint corresponding to the power grid before and after the initial fault, respectively; $\boldsymbol{x}$ is the state variable.

3.3.2. Outer Layer Optimization Model. The outer layer optimization is based on the inner layer model to solve the power grid safety margin. The objective function is to maximize the power grid safety margin and minimize the power generation operation cost. The expression of the objective function is as follows:

$$
\left\{\begin{array}{l}
F_{2}=\max \left(F_{1}\right) \\
F_{3}=\min (C)
\end{array}\right.
$$

where $F_{2}$ and $F_{3}$ are the objective functions of the outer model; $F_{1}$ is the power grid safety margin calculated by the inner model; $C$ is the power generation operation cost.

\section{Constraints:}

(1) Safety constraints of the power grid

According to the previous analysis, under the action of $S$, when the initial fault $L_{a}$ is cut off, the power grid is in a safe state without cascading fault. The conditions are as follows:

$$
I=\min \left(I_{b}\right)>0 .
$$

(2) Power flow constraints

In the safe state, the power grid also needs to meet certain power flow constraints. The constraints similar to formula (8) are given, which can be expressed as

$$
\begin{cases}\text { s.t. } & d^{0}(\mathbf{x})=0 \\ & d^{1}(\mathbf{x})=0 \\ & h^{0}(\mathbf{x}) \geq 0 \\ & h^{1}(\mathbf{x}) \geq 0\end{cases}
$$

where $d^{0}$ and $d^{1}$ are the reduced form of the power flow equation constraint corresponding to the power grid before and after the initial fault, respectively; $h^{0}$ and $h^{1}$ are the reduced form of the power flow inequality constraint corresponding to the power grid before and after the initial fault, respectively.

\section{Preventive Control Strategies under Different Initial Fault Scenarios}

Cascading faults often start with an initial fault and then trigger subsequent faults. Therefore, it is important to identify the vulnerable branches in the system and to preventive control. In this paper, based on the power network topology and operation state, we give the definition and evaluation methods of vulnerability indicators, further filter out the important initial faults according to the safety margin, and then propose the preventive control strategies for different initial fault scenarios.

\subsection{Selection of Initial Fault}

\subsubsection{Initial Fault Preselection Based on Vulnerability}

(1) Severity Index. According to the previous analysis, without considering the uncertainty of impedance relay operation and other blocking conditions, the severity of the initial fault can be effectively evaluated from the branch level by the remaining system branches subjected to the initial fault removal. Let the number of the initial faults in the power grid be $a$. The severity index shown in formula (12) can be obtained after further processing of formula (1) as

$$
k_{(a)}=w_{a}\left(\max \left(\frac{\left|I_{i}^{b . s e t}\right|}{\left|I_{i}^{b . m}\right|}\right)\right)+w_{b}\left(\max \left(\frac{\left|I_{j}^{b . s e t}\right|}{\left|I_{j}^{b . m}\right|}\right)\right),
$$

where $w_{a}$ and $w_{b}$ are weights; the value of weights are set as $\omega_{a}=w_{b}=1$, which represents that the branch $i$-side and $j$ side are of equal importance [16]. $k_{(a)}$ integrates the distance protection on both sides, and the maximum disturbance degree on both sides of the remaining branch is defined as the severity index of the initial fault.

(2) Weighted Power Flow Entropy Index. Starting from the level of branch anti-impact ability and system power flow transfer, the branch load rate is used to weight the power flow entropy index. The weighted power flow entropy index considers the influence of branch load rate and the total number of branches on the system operation state, which overcomes the problem that the traditional model only considers the balance of transfer power flow distribution. Define the weighted power flow entropy $V_{(a)}$ after branch $L_{a}$ is cut off as [17]

$$
V_{(a)}=\frac{\left|\sum_{b=1}^{l} \alpha_{a b} \beta_{a b} \ln \beta_{a b}\right|}{\ln l},
$$

where $\beta_{a b}$ is the power flow impact rate on branch $L_{b}$ after branch $L_{a}$ is cut off; $\alpha_{a b}$ is the load rate of branch $L_{b}$ after branch $L_{a}$ is cut off.

(3) Comprehensive Vulnerability Index. To more comprehensively and reasonably evaluate the vulnerability of initial fault, this paper further considers the system efficiency index $S_{(a)}$ [18], branch dielectric $B_{(a)}$ [19], and voltage offset index $U_{(a)}[20]$. After normalizing and weighting each index, the comprehensive vulnerability index $F_{(a)}$ is defined as

$$
F_{(a)}=w_{1} k_{(a)}^{\prime}+w_{2} V_{(a)}^{\prime}+w_{3} S_{(a)}^{\prime}+w_{4} B_{(a)}^{\prime}+w_{5} U_{(a)}^{\prime},
$$

where $k_{(a)}^{\prime}, V_{(a)}^{\prime}, S_{(a)}^{\prime}, B_{(a)}^{\prime}$, and $U_{(a)}^{\prime}$ are the normalized values of each index, respectively. $w_{1}, w_{2}, w_{3}, w_{4}$, and $w_{5}$ are the weights of each index, and the weight is 1 . The selection of each index weight in the practical application can be determined according to field experience. The comprehensive vulnerability index focuses on identifying the branch that is prone to overload in the process of fault propagation and has a serious impact on the topology and operation state of the power grid after removal. The greater the value of $F_{(a)}$, the more likely it is 
to cause cascading faults after fault and the greater the impact on the stable operation of the power grid.

(4) Vulnerability Assessment Methods. According to the above analysis, this paper takes each vulnerability index as the character input and plans to use a fuzzy C-means algorithm (FCM) to effectively divide the branches. FCM algorithm is a clustering algorithm based on classification. It obtains the membership degree of each sample to all class centers by optimizing the objective function, determining the category of samples, to realize the automatic classification of sample data [21]. The objective function I and constraints are as follows:

$$
\left\{\begin{array}{l}
Z=\sum_{O=1}^{w e} \sum_{v=1}^{v e}\left[a_{v}\left(t_{O}\right)\right]^{b}\left(\left\|t_{O}-c_{v}\right\|\right)^{2}, \\
\sum_{O=1}^{w e} \sum_{v=1}^{v e}\left[a_{v}\left(t_{O}\right)\right]^{b}=1,
\end{array}\right.
$$

where $t_{o}$ is the characteristic input quantity composed of each evaluation index; $c_{v}$ is the cluster center of class $v$; $a_{v}\left(t_{o}\right)$ is the membership function of the O-th sample data for class $v ; b$ is the exponential factor of membership $a_{v}\left(t_{o}\right)$; we is the number of samples; ve is the number of sample classifications. The value of the classification number is determined by referring to the classification entropy proposed in the document [22]. The classification entropy $H$ is defined as

$$
H=-\frac{1}{w e} \sum_{O=1}^{w e} \sum_{v=1}^{v e}\left[a_{v}\left(t_{O}\right)\right] \ln \left[a_{v}\left(t_{O}\right)\right],
$$

where the definition of each variable is the same as that in formula (15), and the number with the smallest classification entropy is the best classification number.

The process of selecting the initial fault based on the FCM algorithm is as follows:

(1) Initialize the membership function and clustering center, and determine the algorithm parameters and feature input. The feature input includes the normalized values of the above six evaluation indexes.

(2) The Euclidean distance from each sample to the cluster center $\left\|t_{O}-c_{v}\right\|$ and the classification entropy $H$ of the sample are calculated.

(3) Determine the sample classification number ve and calculate the objective function $Z$. When $\Delta Z$ is less than the given threshold, the calculation terminates and the clustering result is output. Otherwise, return to step 2 to continue the calculation.

In this way, the FCM algorithm can synthesize various vulnerability indexes, preliminarily screen out more important initial faults, and select these initial faults into the expected initial fault set $G_{1}$.

4.1.2. Initial Faults Are Further Selected according to the Safety Margin. According to the selected expected initial fault set $G_{1}$, the safety margin of the power grid corresponding to each initial fault is calculated separately, whereby the safety level of the power grid for cascading faults after each initial fault is cut can be observed. Further, the initial fault with a low safety margin value is selected into the key initial fault set $G_{2}$, which can be expressed as

$$
G_{2}=\left\{L_{a} \mid R_{a}<\varepsilon\right\},
$$

where $\varepsilon$ is the threshold value for screening and $\varepsilon$ is a positive number, which can be given according to experience; $R_{a}$ is the power grid safety margin corresponding to any initial fault branch $L_{a}$. Any initial fault branch can be regarded as a key initial fault as long as it satisfies formula (17).

\subsection{Preventive Control Model for Different Initial Fault} Scenarios. Due to the large scale of the actual power grid, a large number of branches, it leads to a significant reduction in the computational speed of the algorithm. In this paper, the key initial fault set $G_{2}$ is selected based on the power grid vulnerability and safety margin, and preventive control is taken only for the initial faults in $G_{2}$, which overcomes the problem of excessive computation due to previous control strategies that do not screen the initial faults.

For the key initial fault set $G_{2}$, this paper mainly considers the preventive control strategy in two cases: (1) the safety margin and economic cost after the cutoff of each key initial fault are calculated according to the basic model of preventive control respectively; (2) the second case is to transform the basic model of preventive control into an extended model, which applies to all key initial faults in $G_{2}$, its objective function remains unchanged, and the corresponding constraints change from a single initial fault to multiple initial faults. The extended model can be shown as follows:

$$
\begin{cases}F_{1}=\min \left(R_{1}, R_{2}, \ldots, R_{e}\right) & \\ F_{2}=\max \left(F_{1}\right), & F_{3}=\min (C) \\ m_{e}^{0}(\mathbf{x})=0, & m_{e}^{1}(\mathbf{x})=0 \\ g_{e}^{0}(\mathbf{x}) \geq 0, & g_{e}^{1}(\mathbf{x}) \geq 0 \\ d_{e}^{0}(\mathbf{x})=0, & d_{e}^{1}(\mathbf{x})=0 \\ h_{e}^{0}(\mathbf{x}) \geq 0, & h_{e}^{1}(\mathbf{x}) \geq 0 \\ I \geq 0, & e=1,2, \ldots, E\end{cases}
$$

where $e$ is the $e$-th initial fault; $E$ is the number of initial faults; the corresponding constraints are expanded into $E$ sets, and each set of constraints corresponds to one initial fault; the objective function of the inner model is the minimum value of the power grid safety margin $F_{1}$ under multiple initial faults, while the objective function of the outer model is the same as the basic preventive control model. After the preventive control is carried out, it is necessary to ensure that the nodal injection power of the power grid simultaneously satisfies the condition of high safety margin and low economic cost in case of fault of any branch in the key initial fault set $G_{2}$. 


\section{Solution of the Model}

In this paper, we solve the preventive control model, the inner model uses particle swarm optimization (PSO) [23], and the outer model uses a multiobjective particle swarm optimization based on adaptive grid algorithm (AGAMOPSO) [24]. In the solution process, the key variables and results between the inner and outer layers will be transferred to each other as needed, and the specific transfer method is as follows: the inner layer model passes the power grid safety margin to the outer layer model, and the outer layer model optimizes the generator nodal injection power taking into account the power grid safety margin and economic cost and returns the optimized nodal injection power to the inner layer model; then the inner layer model searches for the cascading fault again based on this result. Then, the inner model searches for the critical state of the cascading faults again based on the results to obtain the new power grid safety margin; finally, through the optimization iterations of the inner and outer layers, the optimal generator output is solved to coordinate safety and economy [25].

5.1. Particle Update Criterion. In PSO and AGA-MOPSO algorithms, the position and rate of each particle are updated according to the following formula [24]:

$$
\left\{\begin{array}{l}
y_{i}(t+1)=y_{i}(t)+v_{i}(t+1), \\
v_{i}(t+1)=w v_{i}(t)+c_{1} r_{1}\left(y_{\mathbf{p} . i}-y_{i}(t)\right)+c_{2} r_{2}\left(y_{g}-y_{i}(t)\right),
\end{array}\right.
$$

where $y_{i}(t+1)$ and $v_{i}(t+1)$ are the position and rate of particle $i$ at the $(t+1)$ st time, respectively, where the particle position $y_{i}$ of the inner model corresponds to the nodal injection power vector in the critical state of cascading fault. The particle position $y_{i}$ of the outer model corresponds to the nodal injection power vector of the current state of the power grid; $y_{p . i}$ is the best individual position of particle $i ; y_{g}$ is the best position of the whole particle swarm; $w$ is the inertia coefficient; $c_{1}$ and $c_{2}$ are acceleration factors; $r_{1}$ and $r_{2}$ are random numbers within $[0,1]$.

5.2. Fitness Function. In this paper, the penalty function is introduced to further deal with the constraint problem in the model [26]. The specific process of this method is as follows: in the inner model, $I=0$ in formula (7) is written as $z(\mathbf{x})=0$, and all power flow equality constraints and inequality constraints in formula (8) are written as $m(\mathbf{x})=0$ and $g(\mathbf{x}) \geq 0$, respectively. In the outer model, $I>0$ in formula (10) is written as $p(\mathbf{x})>0$, and all power flow equality constraints and inequality constraints in formula (11) are written as $d(\mathbf{x})=0$ and $h(\mathbf{x}) \geq 0$, respectively. Add the penalty factor, and use the objective function and constraint function to jointly construct the fitness function as follows:

$$
\left\{\begin{aligned}
f a & =F_{1}+M_{1} \sum_{e}\left[\min \left(0, g_{e}(\mathbf{x})\right)\right]^{2}+M_{2} \sum_{e}\left[\min \left(0, m_{e}(\mathbf{x})\right)\right]^{2} \\
& +M_{3}[\min (0, z(\mathbf{x}))]^{2} \\
f b & =\frac{1}{F_{2}}+M_{1} \sum_{e}\left[\min \left(0, h_{e}(\mathbf{x})\right)\right]^{2}+M_{2} \sum_{e}\left[\min \left(0, d_{e}(\mathbf{x})\right)\right]^{2} \\
& +M_{3}[\min (0, p(\mathbf{x}))]^{2} \\
f c & =F_{3}+M_{1} \sum_{e}\left[\min \left(0, h_{e}(\mathbf{x})\right)\right]^{2}+M_{2} \sum_{e}\left[\min \left(0, d_{e}(\mathbf{x})\right)\right]^{2} \\
& +M_{3}[\min (0, p(\mathbf{x}))]^{2}
\end{aligned}\right.
$$

where $f a$ is the fitness function of the inner model; $f b$ and $f c$ are the fitness functions of the outer model; $F_{1}$ is the objective function of the inner model; $F_{2}$ and $F_{3}$ are the objective functions of the outer model; $M_{1}, M_{2}$, and $M_{3}$ are penalty factors; $g_{e}(\mathbf{x})$, $m_{e}(\mathbf{x}), d_{e}(\mathbf{x})$, and $h_{e}(\mathbf{x})$ are some components of $g(\mathbf{x}), m(\mathbf{x}), d(\mathbf{x})$, and $h(\mathbf{x})$, respectively. 


\subsection{Improvement of the Solution Algorithm}

5.3.1. Power Flow Transfer Method. When solving the power flow, the Newton Raphson method is proposed to solve the power flow equation in the form of $m^{1}(\mathbf{x})=0$, to obtain more accurate calculation results. For the power flow equation in the form of $m^{0}(\mathbf{x})=0$, considering that each particle is generated based on $S_{0}$, and the solution of the power flow equation in the form of $m^{0}(\mathbf{x})=0$ under $S_{0}$ is generally known, we should try to use the power flow solution corresponding to $S_{0}$ to obtain the power flow equation in the form of $m^{0}(\mathbf{x})=0$ contained by any particle, to improve the calculation efficiency. Therefore, the following calculation ideas are designed in this paper.

For ease of explanation, the power flow equation in the form of $m^{0}(\mathbf{x})=0$ is defined as [27]

$$
f_{0}(\mathbf{x})=\mathbf{S}
$$

where $\mathbf{S}$ is the nodal injection power of the power grid, $f_{0}$ is the mapping relation corresponding to the power flow equation of the form $m^{0}(\mathbf{x})=0$, and $\boldsymbol{x}$ is the state variable corresponding to $\mathbf{S}$. Based on the previous description, it is clear that there exists $f_{0}\left(\mathbf{x}_{0}\right)=\mathbf{S}_{0}$, where $\mathbf{x}_{0}$ is the state variable under the current initial operating conditions of the power grid, and setting $\mathbf{S}-\mathbf{S}_{0}=\Delta \mathbf{S}, \mathbf{x}-\mathbf{x}_{0}=\Delta \mathbf{x}$, it can be defined as

$$
\Delta \mathbf{x} A_{0}=\Delta \mathbf{S}
$$

where $A_{0}=\left.f^{\prime}(\mathbf{x})\right|_{\mathbf{x}=\mathbf{x}_{0}}$ is the Jacobian matrix. It can be further defined as

$$
\mathbf{x}=\mathbf{x}_{0}+A_{0}^{-1} \Delta \mathbf{S}
$$

Thus, for each particle, according to the value of its nodal injection power $S$, the solution of the tidal equation of the form of $m^{0}(\mathbf{x})=0$, for each particle in the iterative process, can be found using formula (23).

5.3.2. Adaptive Mutation Operation. To prevent the algorithm from falling into local optimum, this paper draws on the idea of literature [28] and introduces an adaptive mutation operation to mutate the current particles with a certain probability to achieve the goal of breaking up the overly aggregated particles, to search a new direction again and increase the chance of finding the global optimal solution. The specific mutation method is as follows.

Calculate the rate of mutation $P_{a}$ :

$$
P_{a}=\frac{1-(\text { iter }-1)}{(\operatorname{Maxt}-1)^{(1 / m t)}},
$$

where iter is the number of current iterations; Maxt is the maximum number of iterations; $m t$ is the coefficient of mutation, and its value is taken as 0.1 .

Then calculate the mutation interval:

$$
\left[\min \left(v_{\min }, y_{i}(t+1)-\Delta y\right), \max \left(v_{\max }, y_{i}(t+1)+\Delta y\right)\right] \text {, }
$$

where $v_{\min }$ and $v_{\max }$ are particle velocity limits; $\Delta y$ can be defined as

$$
\Delta y=P_{a} \times\left(v_{\max }-v_{\min }\right) .
$$

Finally, execute the mutation operation, and the new variable $\boldsymbol{Y}$ can be defined as

$$
\begin{aligned}
Y= & \text { unifrnd }\left[\min \left(v_{\min }, y_{i}(t+1)-\Delta y\right),\right. \\
& \left.\cdot \max \left(v_{\max }, y_{i}(t+1)+\Delta y\right)\right],
\end{aligned}
$$

where unifrnd is a random array function uniformly distributed on the mutation interval.

5.3.3. Choosing the Compromise Optimal Solution. After obtaining a set of Pareto optimal front (POF), the decisionmaker can choose the corresponding compromise optimal solution according to the demand. There are many methods to choose the compromise optimal solution, such as the decision-making method based on membership function [29], the decision-making method based on distance evaluation index [30], and the comprehensive decision-making method based on the combination of FCM clustering and GRP [31, 32]. Because the decision-makers' judgment on the weight of each objective function is uncertain, they often select the compromise optimal solution that can meet each objective function to the greatest extent in the Pareto solution set according to the membership function. In this paper, the membership degree of the Pareto solution is calculated by using fuzzy set theory. The membership degree $\lambda_{r}^{m}$ of Pareto solution in a one-dimensional objective function can be expressed as

$$
\lambda_{r}^{m}= \begin{cases}1, & f_{r}^{m} \leq f^{m \cdot \min } \\ \frac{f^{m \cdot \max }-f_{r}^{m}}{f^{m \cdot \max }-f^{m \cdot \min },} & f^{m \cdot \min }<f_{r}^{m}<f^{m \cdot \max }, \\ 0 & f_{r}^{m} \geq f^{m \cdot \max }\end{cases}
$$

where $f_{r}^{m}$ is the m-dimensional objective function value of the $r$-th optimal solution, $m=\{1,2, \ldots, N\} ; f^{m \text {.min }}$ and $f^{m \cdot \max }$ are the minimum and maximum values of the m-dimensional objective function value, respectively.

The standardized membership degree calculation formula of each Pareto solution is

$$
\lambda_{r}=\frac{\sum_{m=1}^{N} \lambda_{r}^{m}}{\sum_{r=1}^{M} \sum_{m=1}^{N}} \lambda_{r}^{m},
$$

where $M$ is the number of Pareto solutions, $\lambda_{r}$ is the comprehensive membership of the $r$-th Pareto solution, and the solution with the largest $\lambda_{r}$ is selected as the optimal solution.

5.4. Algorithm Flow. Based on the PSO algorithm and AGAMOPSO algorithm, this paper solves the double-layer optimized preventive control model. The overall algorithm flow is shown in Figure 2: 


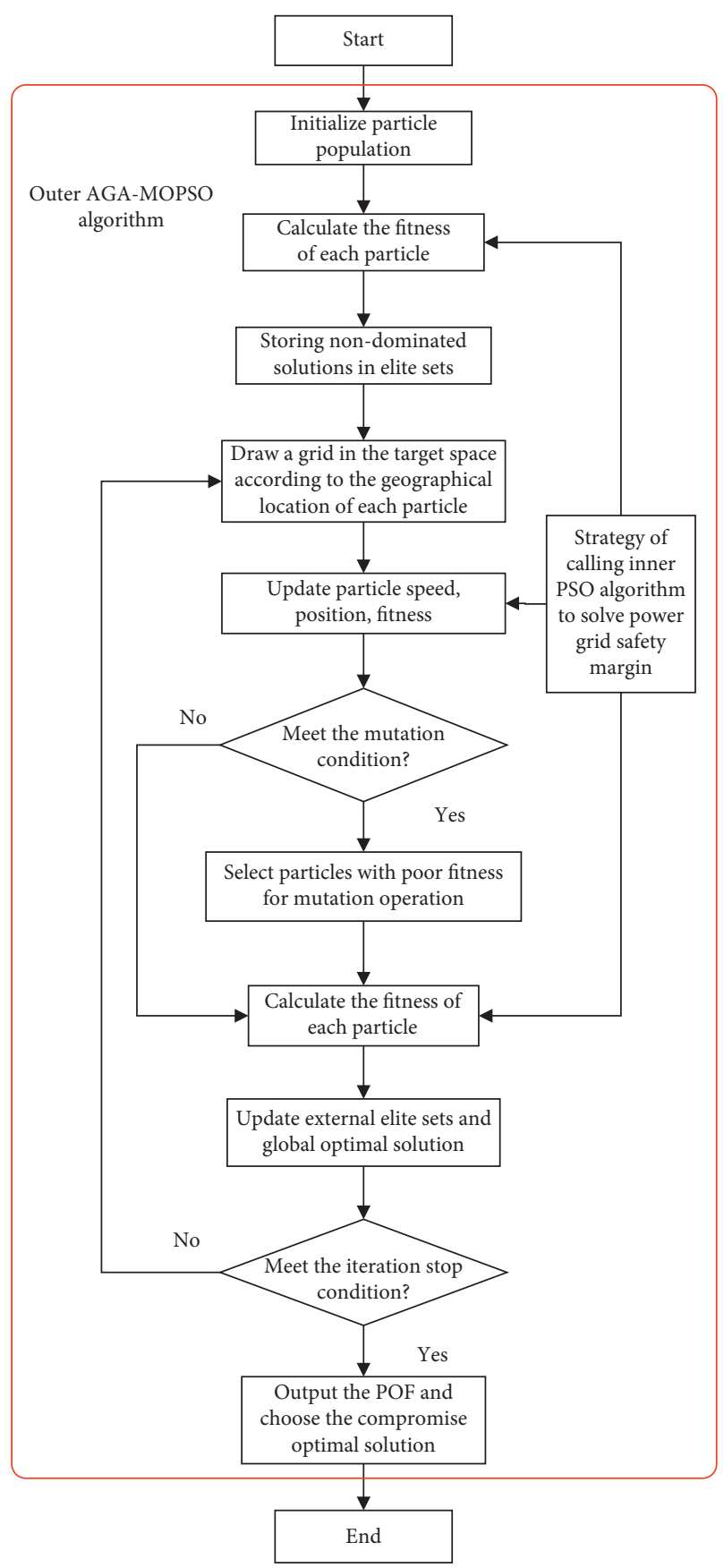

FIgURE 2: Algorithm flowchart.

\section{Example Analysis}

The test example takes the IEEE39 node system as an example, and the system wiring is shown in Figure 3.

According to the system wiring diagram shown in Figure 3, the analysis program is written in the Matlab environment according to the above algorithm. In the following calculation examples, the cost coefficients of each generator of the system can be given concerning literature [15], and the nodal injection power $S_{0}$ corresponding to the parameters of each component of the system and the current power grid operation state can be given concerning literature
[33]. To facilitate the calculation, this paper uses the unit value for representation. For the specific protection configuration data on the IEEE39 node system, this paper uses virtual data and sets the current protection setting value of each branch as three times the current of the branch in the base-state power flow state.

6.1. Initial Fault Analysis. Since the power flow does not converge immediately after the initial fault branch $L_{16-19}$ (the branch between node 16 and node 19, etc.) is cut off, it indicates that there may be voltage instability in the system after the fault, which should be studied in combination with the analysis and control of voltage stability, so branch $L_{16-19}$ is not considered as the initial fault for the time being.

For each index weight in formula (14), this paper determines the weight according to the entropy weight method [34], and the final calculation results are $w_{1}=0.3566$, $w_{2}=0.0561, w_{3}=0.1354, w_{4}=0.2047$, and $w_{5}=0.2472$. Select other nongenerator outlet branches $n-1$ except branch $L_{16-19}$ as the initial fault, normalize each index according to the FCM algorithm process, and then cluster it. Finally, the initial fault is divided into two categories. The clustering centers are shown in Table 1.

From Table 1, it can be seen that the indexes of class A are larger, so the branch of class $A$ is selected into the expected initial fault set $G_{1}$. There are 7 branches in class A. The vulnerability indexes are sorted by size, and the results are shown in Table 2.

As can be seen from Table 2, there is a big difference between the ranking of each vulnerability index individually and the ranking of the integrated vulnerability index $F_{(a)}$, and the branch with the top ranking of the integrated vulnerability index often contains both strong and weak vulnerability indexes, which indicates that the use of a single index for branch vulnerability evaluation is not comprehensive enough and may ignore some weak links, while the integrated vulnerability index takes into account both the network topology and the branch after opening and breaking. The comprehensive vulnerability index takes into account both the network topology and the operation status of the branch after opening and closing, which has certain superiority. To verify the rationality and effectiveness of the initial fault screening, ranking results of the comprehensive vulnerability index of the branch are compared with literature $[35,36]$, and the comparison results show that more than half of the identified vulnerable branches are the same, which indicates that the expected initial fault set $G_{1}$ obtained in this paper is more reasonable and comprehensive.

To further select the initial faults, the safety margin after cutoff of each initial fault in the expected initial fault set $G_{1}$ is calculated separately, and then the conditions are judged to be satisfied according to formula (17), where the value of $\varepsilon$ is taken as 1 . The final calculation results are shown in Table 3.

It can be seen from Table 3 that the safety margins of the initial fault branches $L_{15-16}$ and $L_{21-22}$ after cutoff are less than 1, which meets the conditions for screening key initial faults. Therefore, branches $L_{15-16}$ and $L_{21-22}$ are selected into the key initial fault set $G_{2}$, and preventive control will be taken later. 


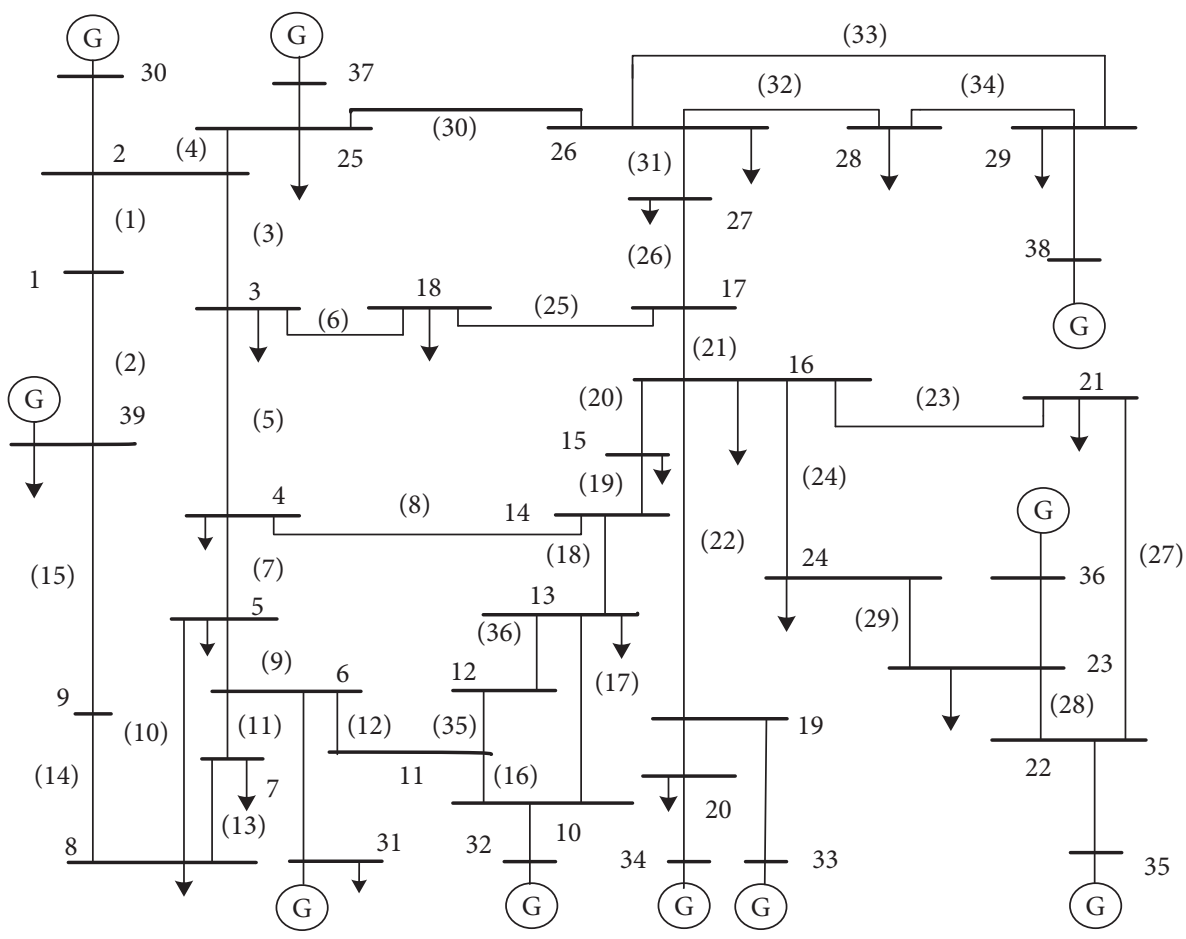

FIgURE 3: Diagram of IEEE39 node system.

Table 1: Cluster center of the example.

\begin{tabular}{lccccrr}
\hline Category & $F_{(a)}$ & $k_{(a)}^{\prime}$ & $V_{(a)}^{\prime}$ & $S_{(a)}^{\prime}$ & $B_{(a)}^{\prime}$ & $U_{(a)}^{\prime}$ \\
\hline A & 0.51 & 0.39 & 0.63 & 0.62 & 0.58 & 0.53 \\
B & 0.16 & 0.06 & 0.37 & 0.29 & 0.17 & 0.16 \\
\hline
\end{tabular}

TABLE 2: Vulnerable branches of IEEE39 node system.

\begin{tabular}{lcccccccccccc}
\hline Branch & $F_{(a)}$ & Ranking & $k_{(a)}^{\prime}$ & Ranking & $V_{(a)}^{\prime}$ & Ranking & $S_{(a)}^{\prime}$ & Ranking & $B_{(a)}^{\prime}$ & Ranking & $U_{(a)}^{\prime}$ & Ranking \\
\hline$L_{15-16}$ & 0.75 & 1 & 0.46 & 3 & 0.73 & 3 & 0.72 & 4 & 0.96 & 2 & 1 \\
$L_{26-27}$ & 0.67 & 2 & 0.58 & 1 & 0.50 & 5 & 0.64 & 5 & 0.32 & 4 & 0.32 \\
$L_{21-22}$ & 0.65 & 3 & 0.39 & 5 & 1 & 1 & 0.31 & 7 & 1 & 1 & 0.84 \\
$L_{2-3}$ & 0.55 & 4 & 0.47 & 2 & 0.74 & 2 & 0.81 & 3 & 0.78 & 3 & 0.32 & 5 \\
$L_{2-25}$ & 0.37 & 5 & 0.45 & 4 & 0.57 & 4 & 0.88 & 2 & 0.19 & 5 & 0.09 & 7 \\
$L_{14-15}$ & 0.33 & 6 & 0.01 & 7 & 0.27 & 7 & 0.60 & 6 & 0.09 & 7 & 0.88 \\
$L_{16-17}$ & 0.32 & 7 & 0.11 & 6 & 0.40 & 6 & 1 & 1 & 0.12 & 6 & 0.43 & 4 \\
\hline
\end{tabular}

TABLE 3: Safety margin for each initial failure.

\begin{tabular}{llllllll}
\hline Initial fault & $L_{14-15}$ & $L_{16-17}$ & $L_{2-3}$ & $L_{26-27}$ & $L_{2-25}$ & $L_{15-16}$ & $L_{21-22}$ \\
\hline
\end{tabular} \begin{tabular}{llllllll}
\hline Safety margin & 3.35 & 2.63 & 1.74 & 1.39 & 1.29 & 0.27 & 0.19
\end{tabular}

6.2. Analysis of Preventive Control Results. In this paper, we study preventive control, and we do not know which branch is going to have cascading faults, so we cannot know which initial fault to give the optimization result by. Therefore, it is necessary to make the preventive control method applicable to all key initial faults, and based on obtaining the key initial fault set $G_{2}$, we consider the preventive control strategies under two different initial fault scenarios, solve the Pareto solutions for the initial fault branches $L_{15-16}$ and $L_{21-22}$ in $G_{2}$ according to the basic preventive control model and the extended preventive control model, respectively, and record the generator node power corresponding to each Pareto optimal solution. Set PSO and AGA-MOPSO algorithm parameters: the maximum number of iterations is 200 , the number of populations is 50, the acceleration factors $c_{1}$ and $c_{2}$ are 2 , the inertia coefficient $w$ is 0.5 , the penalty factors $M_{1}$ and $M_{2}$ are 1000 , and $M_{3}$ is 10000 . The Pareto optimal front (POF) obtained under different initial fault scenarios is as follows.

Figures 4 and 5 are the Pareto solutions calculated according to the basic preventive control model for the initial fault branches $L_{15-16}$ and $L_{21-22}$, respectively, and Figure 6 is the Pareto solutions obtained according to the 


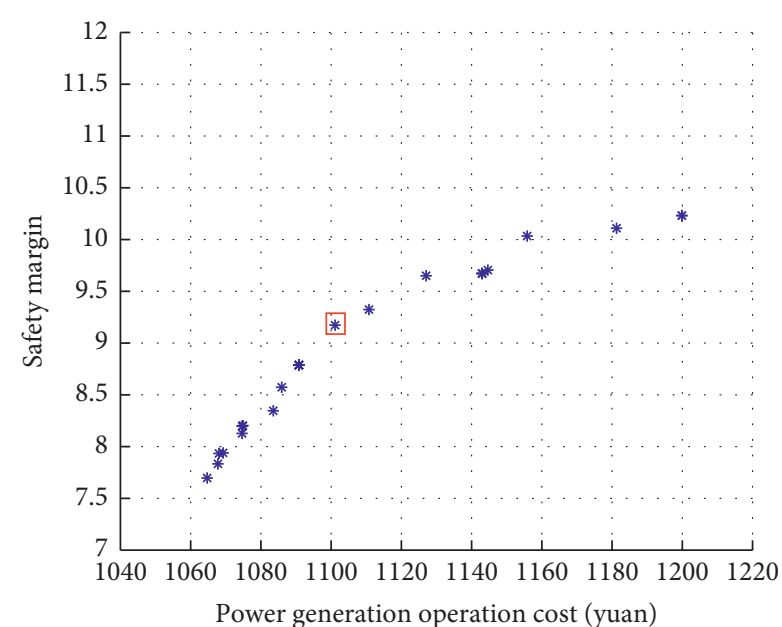

* POF

$\square$ Compromise solution

FIgUre 4: POF from initial failure $L_{15-16}$.

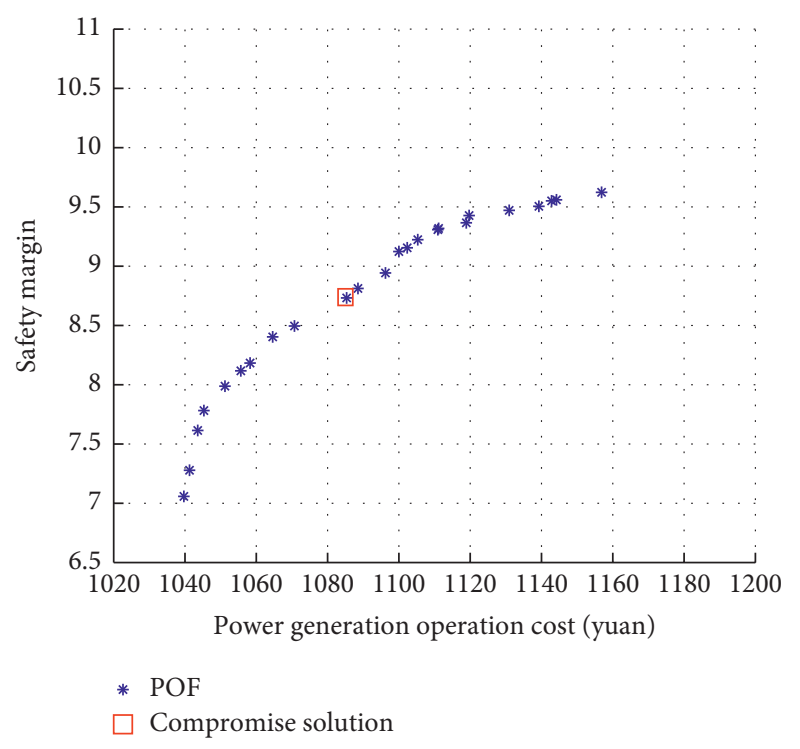

FIGURE 5: POF from initial failure $L_{21-22}$.

extended preventive control model for the initial fault branches $L_{15-16}$ and $L_{21-22}$ at the same time. From the simulation results, it can be seen that the extended model established in this paper is suitable for all initial faults screened, can meet the conditions of high safety margin and low economic cost under any fault, and ensure that the controlled power grid is in a safer operation state. The Pareto solution obtained by the algorithm forms a POF curve. The POF curve has good uniform ductility. Each Pareto solution has a different economic cost and power grid safety margin. The POF curve is from left to right, and the safety margin and economic cost of the corresponding Pareto solution are from small to large. The membership degree of each Pareto solution is calculated through the membership function, and the respective compromise optimal solution is found according to the value of the comprehensive membership

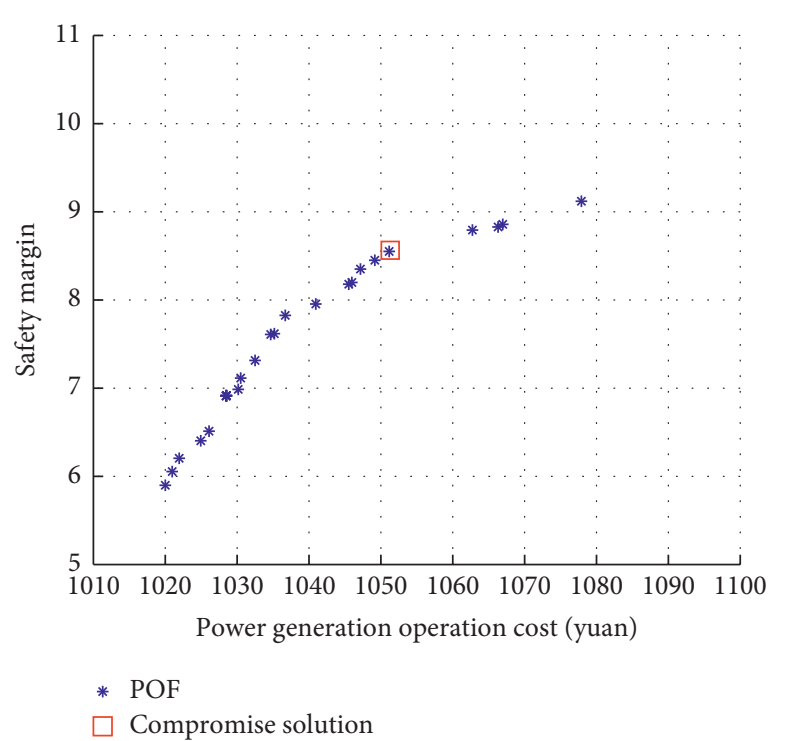

FIgURE 6: POF from initial failures $L_{15-16}$ and $L_{21-22}$ at the same time.

TABle 4: Preventive control optimization results.

\begin{tabular}{lccc}
\hline Generator no. & $L_{15-16}$ & $L_{21-22}$ & $L_{15-16} ; L_{21-22}$ \\
\hline G30 & 881.93 & 899.31 & 477.33 \\
G32 & 634.51 & 581.78 & 166.43 \\
G33 & 168.36 & 389.95 & 639.32 \\
G34 & 250.43 & 558.72 & 506.11 \\
G35 & 714.05 & 562.74 & 503.09 \\
G36 & 218.75 & 268.34 & 286.76 \\
G37 & 550.46 & 624.76 & 962.73 \\
G38 & 885.92 & 631.39 & 354.63 \\
G39 & 860.50 & 631.85 & 832.60 \\
Safety margin & 9.17 & 8.73 & 8.55 \\
Generation cost & 1101.12 & 1085.34 & 1051.16 \\
\hline
\end{tabular}

degree. The results of the optimal solution are shown in Table 4:

The method given in this paper is the simulation and tested on several other node systems as well, and the results are satisfactory, which shows that the method in this paper is suitable for various systems. From the above calculation example, it is clear that the safety and economy of the power grid are significantly improved after preventive control for all possible key initial fault sets. At this point, the power grid enters a safer operating state $S_{0}$, and when the power grid nodal injection power in an arbitrary variation within a hypersphere with $S_{0}$ as the center of the sphere and the safety margin value $R$ as the radius, the power grid is safe for different key initial fault impacts for the triggering of cascading faults; that is, no cascading faults are triggered. In conclusion, compared with the existing methods, the method in this paper can facilitate the observation of the safety of the power grid and the reasonable variation range of the nodal injection power of the power grid and minimizes the generation cost during the power grid adjustment process, providing the operators with the optimal generator output strategy that balances safety and economy. 


\section{Conclusion}

Considering safety and economy, this paper presents a multiobjective preventive control model and specific solution process for preventing cascading faults of the power grid and verifies it with a numerical example. The main conclusions are as follows:

(1) The preventive control model established in this paper is essentially a double-layer optimization model with dynamic interaction and interaction between internal and external layers. The optimal generator output strategy is obtained by solving the double-layer model so that the power grid can take into account the requirements of safety and economy at the same time.

(2) In this paper, the safety margin of the power grid is mainly measured by the distance between the current operating state and the critical state of the power grid, which is in the form of the distance between the nodal injection power vectors of the power grid in the two states. This form of safety margin representation can visually quantify and compare the safety level of the power grid for cascading faults before and after taking preventive measures.

(3) In this paper, for the selection of initial faults, the selection is mainly made considering the vulnerability indexes of the network topology and operation state as well as the safety margin, ensuring that the important initial faults are selected.

In conclusion, the method proposed in this paper can effectively select the initial faults that are more important to the power grid and preventive control according to the idea of improving the safety margin of the power grid and ensuring the economic cost, providing a reference for further research.

\section{Data Availability}

The data used to support the findings of this study are available from the corresponding author upon request.

\section{Conflicts of Interest}

The authors declare that they have no conflicts of interest.

\section{Acknowledgments}

This research was supported by the Scientific Research Development Foundation of the Fujian University of Technology under Grant no. GY-Z17149, the Scientific and Technical Research Project of Fuzhou under Grant no. GY-Z18058, and the Open Fund of Fujian Provincial University Engineering Research Center under Grant nos. KF-X19016 and KF-D21009.

\section{References}

[1] X. Liu and Z. Li, "Revealing the impact of multiple solutions in DCOPF on the risk assessment of line cascading failure in OPA model," IEEE Transactions on Power Systems, vol. 31, no. 5, pp. 4159-4160, 2016.
[2] S. Shengwei Mei, F. Fei He, X. Xuemin Zhang, S. Gang Wang, and G. Wang, "An improved OPA model and blackout risk assessment," IEEE Transactions on Power Systems, vol. 24, no. 2, pp. 814-823, 2009.

[3] H. Dong and L. Cui, "System reliability under cascading failure models," IEEE Transactions on Reliability, vol. 65, no. 2, pp. 929-940, 2016.

[4] M. Ding, Y. Guo, J. Zhang, and Y. Qian, Y. Qi, J. He, and J. Yi, Node vulnerability assessment for complex power grids based on effect risk entropy-weighted fuzzy comprehensive evaluation," Diangong Jishu Xuebao/Transactions of China Electrotechnical Society, vol. 30, no. 3, pp. 214-223, 2015.

[5] A. E. Da Vid, B. Gjorgiev, and G. Sansavini, "Quantitative comparison of cascading failure models for risk-based decision making in power systems," Reliability Engineering and System Safety, vol. 198, Article ID 106877, 2020.

[6] X. Dong, E. Guan, L. Jing, H. Wang, and S. Mirsaeidi, "Simulation and analysis of cascading faults in hybrid AC/DC power grids," International Journal of Electrical Power and Energy Systems, vol. 115, Article ID 105492, 2020.

[7] F. B. Ajaei and R. Iravani, "Dynamic interactions of the MMC-HVDC grid and its host AC system due to AC-side disturbances," IEEE Transactions on Power Delivery, vol. 31, no. 3, pp. 1289-1298, 2016.

[8] L. Xue and Z. Qi, "Impact of cascading failure based on line vulnerability index on power grids," Energy Systems, pp. 1-26, 2021.

[9] F. U. Rong, G. P. Jiang, and B. Y. Wang, "A preventive control strategy for power grid considering system cascading failure risk," Power System Protection and Control, vol. 39, no. 3, pp. 12-17, 2011.

[10] J. Ren, J. Wei, and Y. Gu, "Preventive control based on multiobjective particle swarm optimization algorithm for cascading trips," Electric Power Automation Equipment, vol. 36, no. 07, pp. 53-59, 2016.

[11] M. Ding, Y. Qian, J. Zhang, J. He, and J. Yi, "Defence model based on multistage dynamic game with consideration of bounded rationality against power system cascading failure," Dianli Zidonghua Shebei/Electric Power Automation Equipment, vol. 37, no. 2, pp. 69-74+82, 2017.

[12] J. Zhang, Y. Yang, and L. I. Xiaoyan, "Coordinated control model of power system cascading failure considering safety and economy," Proceedings of the CSEE, vol. 38, no. 16, pp. 4784-4791+4983, 2018.

[13] C. P. Yang, R. Yao, and S. Zhang, "Cascading outage risk mitigation method coordinating economy and safety in power systems," Advanced Technology of Electrical Engineering and Energy, vol. 36, no. 12, pp. 71-78, 2017.

[14] D. Huiqiong, L. Qinbinb, W. Junyuan, Z. Rongjin, and L. Peiqian, "Research on the method of observing the safety level of the grid cascading trip based on node injection power," Electric Power Systems Research, vol. 199, 2021.

[15] C. Luo, F. Lin, and L. I. Zi, "Optimal power flow model considering operation risk of cascading failure," Smart Power, vol. 46, no. 1, pp. 57-62, 2018.

[16] H. Li, Y. Luo, and J. Wu, "Collision-free path planning for intelligent vehicles based on bézier curve," IEEE Access, vol. 7, pp. 123334-123340, 2019.

[17] X. U. Yan, J. Y. Wen, and X. L. Zhang, "Critical lines identification in power grid based on vulnerability of line under impact and disconnection consequence," Power System Protection and Control, vol. 48, no. 1, pp. 81-87, 2020.

[18] H. Deng and Y. Zhang, "An algorithm for identifying vulnerability branches in power network based on cascading 
overload tripping mode due to overload," Smart Grid, vol. 3, no. 4, pp. 283-287, 2015.

[19] S. A. Xiong, C. Guo, and H. E. Yubin, "Vulnerable line evaluation in power grid considering line betweenness and well-being risk contribution degree," Electric Power Construction, vol. 36, no. 12, pp. 84-90, 2015.

[20] X. Liu, Y. Mao, S. Liang et al., "Identification of vulnerable lines in power grid based on comprehensive betweenness index," Power System Protection and Control, vol. 44, no. 2, pp. 116-121, 2016.

[21] Q. Xu, L. ü Yajuan, and H. Sun, "Refined analysis of largeconsumers' interruptible features from multi-dimension," Transactions of China Electrotechnical Society, vol. 35, no. S1, pp. 284-293, 2020.

[22] P. de Boves Harrington, "Support vector machine classification trees based on fuzzy entropy of classification," Analytica Chimica Acta, vol. 954, pp. 14-21, 2017.

[23] H.-Q. Deng, J. Luo, K.-C. Chang, Q.-B. Li, R.-J. Zheng, and P.-Q. Li, "Research on security level evaluation method for cascading trips based on WSN," Wireless Communications and Mobile Computing, vol. 2021, Article ID 6649127, 2021.

[24] J. I. Zhen-Ping and J. Yang, "Multi-objective coordinated optimization based on Pareto frontier in continuous casting," Control Engineering China, vol. 25, no. 1, pp. 76-81, 2018.

[25] Y. Li and K. Li, "Incorporating demand response of electric vehicles in scheduling of isolated microgrids with renewables using a Bi-level programming approach," IEEE Access, vol. 7, pp. 116256-116266, 2019.

[26] P. Srivastava and J. Cortés, "Nesterov acceleration for equality-constrained convex optimization via continuously differentiable penalty functions," IEEE Control Systems Letters, vol. 5, no. 2, pp. 415-420, 2021.

[27] X. Wang, Modern power system analysis, Science Press, Beijing, China, 2003.

[28] X. I. Shen, Adaptive Particle Swarm Optimization Algorithm and its Application, Tsinghua University Press, Beijing, China, 2015.

[29] R. Liu, Y. Jia, and K. Fu, "Double-layer optimization for wind and energy storage capacity configuration in transmission network considering safety reserve constraints," Power System Technology, vol. 2021, no. 7, pp. 2741-2752, 2021.

[30] J. Liu and X. Luo, "Environmental economic dispatching adopting multiobjective random black-hole particle swarm optimization algorithm," Proceedings of the CSEE, vol. 30, no. 34, pp. 105-111, 2010.

[31] M. Zhang and Y. Li, "Multi-objective optimal reactive power dispatch of power systems by combining classification-based multi-objective evolutionary algorithm and integrated decision making," IEEE Access, vol. 8, pp. 38198-38209, 2020.

[32] Y. Li, Y. Li, G. Li, D. Zhao, and C. Chen, "Two-stage multiobjective OPF for AC/DC grids with VSC-HVDC: incorporating decisions analysis into optimization process," Energy, vol. 147, pp. 286-296, 2018.

[33] Z. Guan, The Static Voltage Stability Analysis Based on Thevenin Equivalent, Harbin Institute of Technology, Harbin, China, 2019.

[34] N. Zhang and J. Cai, "Evaluation of oil-paper insulation condition based on fuzzy matter element-technique for order preference by similarity to ideal solution method," Transactions of China Electrotechnical Society, vol. 33, no. 22, pp. 5381-5389, 2018.

[35] J. Ren and J. Wei, "Vulnerable line identification and its transmission section search for power system," Electric Power Automation Equipment, vol. 35, no. 7, pp. 37-42, 2015.

[36] L. Zeng, X. Zeng, and L. V. Jia, "Identification of vulnerable line of power grid in view of grid structure and running state," Smart Power, vol. 46, no. 8, pp. 8-12+51, 2018. 